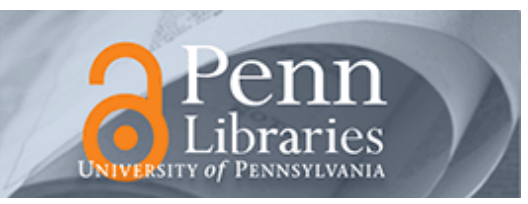

University of Pennsylvania

ScholarlyCommons

Departmental Papers (CBE)

Department of Chemical \& Biomolecular

Engineering

October 2005

\title{
On-lattice kinetic Monte Carlo simulations of point defect aggregation in entropically influenced crystalline systems
}

\author{
Jianguo Dai \\ University of Pennsylvania \\ Joshua M. Kanter \\ University of Pennsylvania \\ Sumeet S. Kapur \\ University of Pennsylvania \\ Warren D. Seider \\ University of Pennsylvania, seider@seas.upenn.edu \\ Talid Sinno \\ University of Pennsylvania, talid@seas.upenn.edu
}

Follow this and additional works at: https://repository.upenn.edu/cbe_papers

\section{Recommended Citation}

Dai, J., Kanter, J. M., Kapur, S. S., Seider, W. D., \& Sinno, T. (2005). On-lattice kinetic Monte Carlo simulations of point defect aggregation in entropically influenced crystalline systems. Retrieved from https://repository.upenn.edu/cbe_papers/60

Copyright American Physical Society. Reprinted from Physical Review B, Volume 72, Issue 13, Article 134102, October 2005, 10 pages.

Publisher URL: http://dx.doi.org/10.1103/PhysRevB.72.134102

This paper is posted at ScholarlyCommons. https://repository.upenn.edu/cbe_papers/60

For more information, please contact repository@pobox.upenn.edu. 


\title{
On-lattice kinetic Monte Carlo simulations of point defect aggregation in entropically influenced crystalline systems
}

\author{
Abstract \\ An on-lattice kinetic Monte Carlo model of vacancy aggregation in crystalline silicon is parametrized \\ using direct regression to evolution data from nonequilibrium molecular dynamics simulations. The \\ approach bypasses the need to manually compute an energy barrier for each possible transition and \\ leads to an excellent, robust representation of the molecular dynamics data. We show that the resulting \\ lattice kinetic Monte Carlo model correctly captures the behavior of the real, continuous space system by \\ properly accounting for continuous space entropic effects, which are often neglected in lattice-based \\ models of atomistic processes. These contributions are particularly important at the high temperatures \\ relevant to many steps in semiconductor materials processing.

\section{Comments} \\ Copyright American Physical Society. Reprinted from Physical Review B, Volume 72, Issue 13, Article \\ 134102, October 2005, 10 pages. \\ Publisher URL: http://dx.doi.org/10.1103/PhysRevB.72.134102
}




\title{
On-lattice kinetic Monte Carlo simulations of point defect aggregation in entropically influenced crystalline systems
}

\author{
Jianguo Dai, Joshua M. Kanter, Sumeet S. Kapur, Warren D. Seider, and Talid Sinno* \\ Department of Chemical and Biomolecular Engineering, University of Pennsylvania, Philadelphia, Pennsylvania 19104, USA
}

(Received 1 June 2005; published 6 October 2005)

\begin{abstract}
An on-lattice kinetic Monte Carlo model of vacancy aggregation in crystalline silicon is parametrized using direct regression to evolution data from nonequilibrium molecular dynamics simulations. The approach bypasses the need to manually compute an energy barrier for each possible transition and leads to an excellent, robust representation of the molecular dynamics data. We show that the resulting lattice kinetic Monte Carlo model correctly captures the behavior of the real, continuous space system by properly accounting for continuous space entropic effects, which are often neglected in lattice-based models of atomistic processes. These contributions are particularly important at the high temperatures relevant to many steps in semiconductor materials processing.
\end{abstract}

DOI: 10.1103/PhysRevB.72.134102

PACS number(s): 61.72.Ji, 61.72.Cc

\section{INTRODUCTION}

The kinetic Monte Carlo (KMC) method has been applied extensively in various forms to the study of microstructural evolution in crystalline materials such as metals and semiconductors. While molecular dynamics (MD) offers a greater degree of resolution, and only requires an interatomic force field as input, it is limited in terms of the system scales that can be addressed (nanoseconds and nanometers). KMC, on the other hand, coarse-grains the details of atomic vibration (the principal time-scale limitation in MD), but retains much of the microscopic morphological information. The principal drawback in $\mathrm{KMC}$ is that mechanistic information must be supplied externally, ${ }^{1}$ as in the case of continuum scale modeling.

There are two main classes of explicit-atom KMC simulations - on-lattice and off-lattice. In the former, all species are restricted to move and interact on a fixed lattice, while in the latter all coordinates are possible. On-lattice KMC (LKMC) offers significant computational advantages because it is much simpler to specify the allowable event database. However, the fixed lattice restriction can lead to serious errors when the microstructural evolution under consideration does not strictly adhere to the lattice. In some cases, this possibility is obvious, such as in the case of interstitial atom diffusion and aggregation, where the interstitial atoms are, by definition, not on lattice sites. In others, it is not possible to predict whether an on-lattice representation is appropriate without more detailed calculations.

The required inputs for on-lattice KMC simulations of aggregation phenomena, which are the focus of this paper, are rates for the various allowable events, such as diffusion, reaction, and clustering. There are several approaches for specifying the rate process database in a lattice KMC simulation. These include (1) full enumeration of all possible transitions, (2) specifying a fixed set of possible events, and (3) using the change in the number of bonds (which are generally defined as the energy of interaction between species on neighboring lattice sites) that result from a transition. The relative merits of each of these approaches have been discussed by Adams et al., ${ }^{2}$ and the literature contains many examples of each (e.g., see Refs. 3-5).

The interaction distance between particles in a KMC simulation is a particularly important parameter. For example, La Magna et al. ${ }^{6}$ used a bond-counting model for vacancy aggregation in $\mathrm{Si}$, which includes interactions up to the second-nearest-neighbor distance. Fichthorn and Scheffler ${ }^{7}$ employed discrete Fourier transform (DFT) calculations to compute pairwise interactions as a function of distance up to the 13th nearest-neighbor (NN) shell in Ag islands on Pt (111). The number of rates that must be specified for a complete description of a system can be large, particularly in cases where long-range interactions exist between particles and multiple chemical species are present. In general, increasing the number of rate processes considered increases the potential accuracy of the KMC simulation, but also increases the number of parameters that must be specified, and concomitantly the computational expense of the algorithm. Reducing, or coarse-graining, the allowable event set is difficult to validate without detailed comparisons to more accurate calculations or experimental data.

The aims of this paper are twofold. The first is to present an alternative approach to on-lattice KMC rate parametrization, which is usually performed using the laborious process of computing energy barriers for every event in the database. In the present work, KMC rates are determined by direct regression to molecular dynamics evolution data. While MD can only access short times, we argue that for certain processes such as the vacancy aggregation studied here, the accessible time scale is sufficient to extract the important rates. The second aim is to highlight some basic issues related to the description of intrinsically continuous-space phenomena onto on-lattice representations. In particular, we highlight how entropic effects, which are often neglected in lattice $\mathrm{KMC}$ models, are captured in the rate parametrization approach discussed here.

In the present work, we use nonequilibrium vacancy clustering in crystalline silicon to demonstrate these very general concepts. This particular system choice is based on the fact that vacancy aggregation is a technologically important pro- 


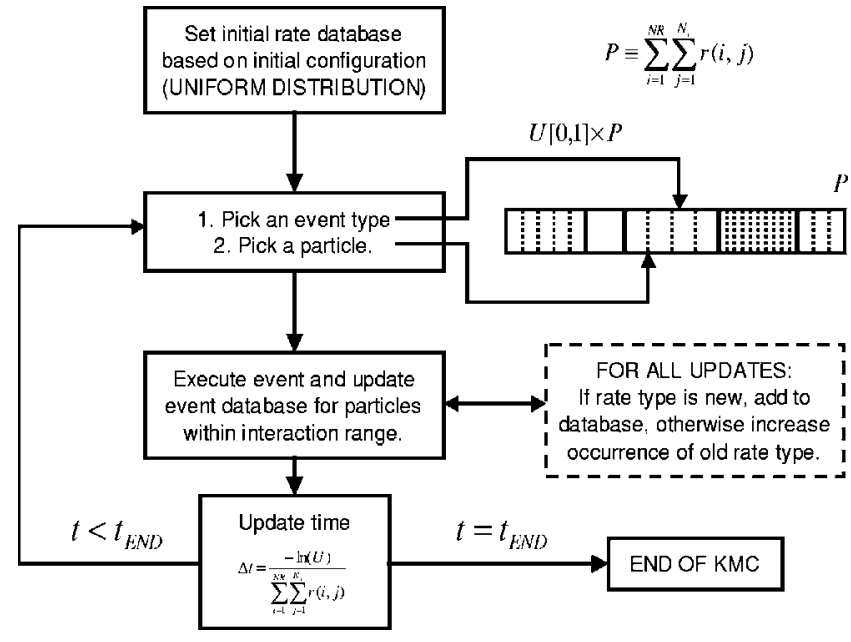

FIG. 1. Schematic representation of KMC algorithm.

cess that has been characterized in detail with experiments ${ }^{8,9}$ and detailed continuum models, ${ }^{10-12}$ and also because accurate empirical interatomic potentials are readily available. ${ }^{13-15}$ Vacancy aggregation is also often assumed to be a prototypical "on-lattice system," an assumption that we show here to be invalid, particularly at elevated temperature. It is expected that the overall approach presented in this paper should be applicable to any system that is dominated by processes that can be captured on the molecular dynamics time scale.

The paper is organized as follows. In Sec. II the KMC algorithm and bonding model for describing the possible event database are presented. The MD-KMC regression is described in Sec. III, along with the MD data that is used for inferring the KMC rates. Results and discussion are presented in Sec. IV, followed by conclusions in Sec. V.

\section{KMC MODEL}

\section{A. Algorithm}

The overall structure of the bond-counting LKMC model used in this study is shown in Fig. 1. The mobile species (vacancies) first are placed uniformly throughout the lattice and the rate matrix is initialized. At each simulation step, all the possible events are grouped into different types according to their rates. Each rate type, therefore, can include multiple (different) events that possess the same rate, thereby reducing the number of unique event types in the simulation. In Fig. 1, NR is the total number of rate types, $N_{i}$ is the number of events of type $i$, and $P$ is the sum of all the rates of the events that are possible at any given time. The standard binary-tree search algorithm is used to pick an event type first based on the total rate, $P$, and a uniform random number, $U \in[0,1]$. Once a rate type is selected, a particular particle is randomly chosen to execute the event (from the relevant subset of particles).

Once the selected event is executed, the local configuration and the rate database for vacancies that are possibly affected by the execution of the event are updated accordingly. The rate database only includes rate types that have been accessed thus far. If a new event type is generated by the previous move, it is added to the database. Finally, the simulation time is updated using $\Delta t=-\ln U / P$ ( $U$ is another uniform random number, $U \in[0,1]$, and the algorithm is repeated until the final time $t_{\text {end }}$ is reached.

\section{B. KMC bonding model}

The bonding model used here to describe the vacancy cluster energies and therefore the transition rates is a generalization of the model developed by La Magna et al. ${ }^{6}$ In their model, La Magna et al. assumed that the vacancy-vacancyinteraction distance extended up to the second-nearestneighbor distance $(2 \mathrm{NN})$. An important feature of their model was a screening effect that prevented interaction "overcounting," whereby two vacancies separated by a lattice site only interact if the shared nearest neighbor (1NN) site does not contain another vacancy. The energy of the entire system is then given by

$$
E_{b}=\sum_{\langle i, j\rangle} \frac{E_{b}^{1}}{2} S_{i} S_{j}+\sum_{\langle l, m\rangle} \frac{E_{b}^{2}\left(S_{k}\right)}{2} S_{l} S_{m},
$$

where $E_{b}$ is the energy of the system and $E_{b}^{1}$ and $E_{b}^{2}$ are the bond energies associated with $1 \mathrm{NN}$ and $2 \mathrm{NN}$ interactions, respectively. The $S_{x=i, j, k, l, m}$ are site occupancy indices: $S_{i}=1$ if site $i$ is occupied by a vacancy, $S_{i}=0$ if it is empty. The screening physics are represented by $S_{k}$, where $k$ is the shared 1NN site between two $2 \mathrm{NN}$ vacancies: $S_{k}=1$ if a shared $1 \mathrm{NN}$ interaction exists that is associated with a pair of $2 \mathrm{NN}$ vacancies on sites $l$ and $m, S_{k}=0$ otherwise, so that

$$
E_{b}^{2}\left(S_{k}\right)= \begin{cases}0 & \text { if } S_{k}=1 \\ E_{b}^{2} & \text { if } S_{k}=0 .\end{cases}
$$

The physical interpretation of the screening physics is discussed below. In the work of La Magna et al., the bonding energies, $E_{b}^{1}$ and $E_{b}^{2}$, were computed by fitting to detailed tight-Binding molecular dynamics (TBMD) calculations of various cluster energies at a fixed temperature. ${ }^{16}$ While the $\mathrm{KMC}$ predictions appear to be qualitatively reasonable, it is difficult to say whether the model is mechanistically and quantitatively correct, at least with respect to the TBMD simulations that were used to parametrize the KMC parameters.

The need for the extended-range bonding model used in the present study is based on the observation from MD simulations that the vacancy-vacancy interaction actually extends significantly beyond the $2 \mathrm{NN}$ distance. In fact, MD simulations with various potentials ${ }^{17,18}$ indicate that the interaction extends up to the 4NN distance along the (110) direction, and somewhat less along other directions. Assuming that the vacancy-vacancy interaction is approximately isotropic, the interaction distance therefore can extend up to the $8 \mathrm{NN}$ interaction shell! In our previous work, ${ }^{11}$ it was further shown that this interaction distance was essentially independent of the local environment, and can be assumed to be constant.

An extended-range bonding model that retains the screening concept discussed above is illustrated in Fig. 2. For any given vacancy pair within interaction distance, the interac- 


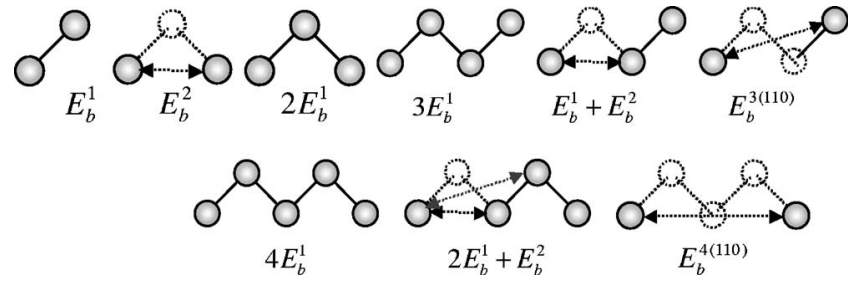

FIG. 2. Examples of bonding energies for various configurations aligned along the (110) crystallographic direction. The notation $E_{b}^{3(110)}$ and $E_{b}^{4(110)}$ indicate a third-nearest neighbor and fourthnearest neighbor interaction along the (110) direction, respectively.

tion is screened if there exist one or more vacancies that are closer to both than they are to each other. The screening volume around two particles is determined by constructing a sphere so that the particles lie on its circumference and are connected by a diameter as shown in Fig. 3. The interaction between vacancies $A$ and $B$ is screened if there are one or more vacancies in the sphere.

The physical motivation for including screening into a bonding model for vacancies is most easily understood by considering that large vacancy clusters in any material, i.e., cavities, are characterized thermodynamically by their surface area, not their volume. ${ }^{19}$ In reality, the driving force for vacancy clustering is the reduction of dangling bonds on atoms in the crystal matrix and not direct affinity between the holes themselves.

Consider, for example, the hexagonal vacancy cluster shown in Fig. 4. Without screening, there are a total of six $1 \mathrm{NN}$, six $2 \mathrm{NN}$, and three $3 \mathrm{NN}$ interactions, leading to a volumetric contribution to the total bonding energy. With the screening model outlined in Figs. 3 and 4 however, there are only six $1 \mathrm{NN}$ interactions, which are clearly representative of the cluster surface area. Interestingly, it will be shown in $\mathrm{Sec}$. IV C that it is not possible to obtain the correct cluster-

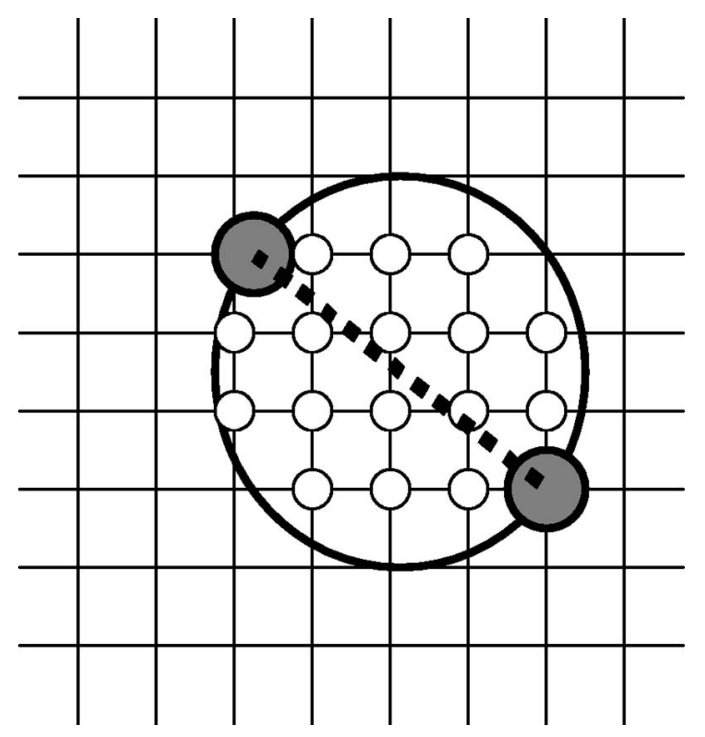

FIG. 3. Two-dimensional representation of screening volume between two vacancies (gray circles). Any occupied lattice sites (small white circles) within the large circle lead to a screening of the interaction between the two vacancies.

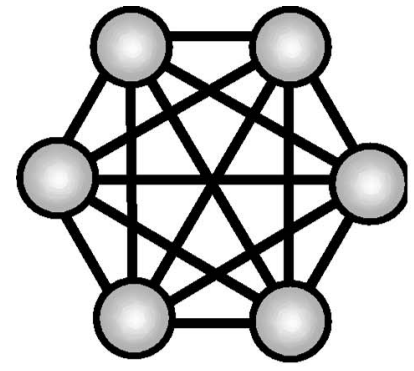

(a)

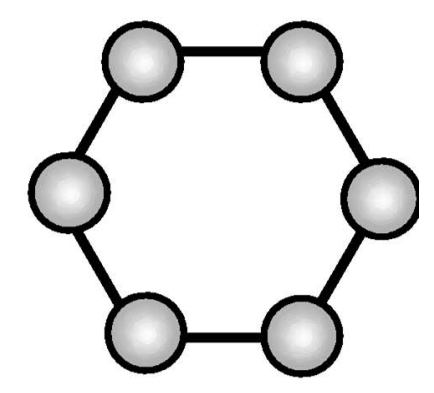

(b)

FIG. 4. Interactions of a hexagonal cluster (a) without and (b) with interaction screening.

ing physics without the screening mechanism, regardless of the values used for the various bonding energies.

\section{Transition barriers and rates for on-lattice simulations}

In principle, transition barriers for all events during the KMC simulation can be computed based on the initial and final states and the energy barrier associated with an isolated single vacancy hop, $\Delta E_{\text {hop }}$, i.e., ${ }^{7}$

$$
\Delta E_{\text {total }}=0.5 \times\left(E_{\text {final }}-E_{\text {init }}\right)+\Delta E_{\text {hop }} .
$$

The energy barrier for any transition is therefore given by

$$
\Delta E_{i}=\max \left(0, \Delta E_{0}-0.5 \times \sum_{j=1}^{N N}\left[\Delta N B_{j} E_{b}^{j}\right]\right),
$$

where $\Delta E_{i}$ is the energy barrier for event $i, N N$ is the maximum interaction shell, $\Delta N B_{j}$ is the bond-count change due to a hop associated with interaction range $j, E_{b}^{j}$ is the corresponding bond energy. Note that all barriers are positive; if the energy difference between the initial and final states is negative and larger in magnitude than $\Delta E_{0}$, no energy barrier exists for the hop. The rate of an event $i$ is then simply given by an Arrhenius expression of the form 


$$
r_{i}=v_{0} \exp \left(\frac{-\Delta E_{i}}{k_{B} T}\right),
$$

where $r_{i}$ is the rate for event $i, v_{0}$ is the overall attempt frequency, $k_{B}$ is the Boltzmann constant, and $T$ is the temperature.

While the $E_{b}^{j}$ can be computed using molecular statics/ dynamics (empirical or electronic structure based), it is important to note some important limitations of lattice-based KMC. In off-lattice Monte Carlo simulations (kinetic or otherwise), changes in the configurational and vibrational entropy between the initial state and final state are implicitly accounted for because the system is being evolved in the full (continuous) coordinate space. In lattice $K M C$, however, atomic moves are performed on a much lower dimensional subspace that neglects all of the vibrational, and most of the configurational, entropy changes associated with a transition. We have recently shown (a) that the configurational entropy associated with vacancy clusters at high temperatures is entirely due to an enormous number of off-lattice configurations, ${ }^{20}$ and (b) this configurational entropy is large enough to substantially alter the equilibrium morphology of these clusters. ${ }^{12}$

Obviously, these off-lattice states cannot be represented explicitly in a lattice model. In some sense, each configuration in the on-lattice system is a representative of a local ensemble of off-lattice configurations in the continuous system. A transition between two on-lattice configurations, therefore, can be considered to be an effective transition between two ensembles. The nature of these ensembles is a function of the degeneracy of the various configurations as well as the distribution of vibrational states associated with each one. Detailed analysis of this concept will be presented in a future publication. Here, we hypothesize that reproducing the vacancy size distribution predicted by MD implies that we have captured these off-lattice characteristics. Equally importantly, we will show that leaving them out leads to severe errors, particularly at high temperature.

\section{KMC MODEL REGRESSION TO MD DATA}

As mentioned in the Introduction, the overall approach taken in this work to determine effective bond energies, $E_{b}^{j}$, is to regress them using direct comparison to MD evolution data. In this section, we first outline the specific MD data that will be used to compute the KMC rates, and then discuss the regression approach.

\section{A. Molecular dynamics evolution data}

A single constant- $N V T$ ensemble MD simulation was performed in which 1000 uniformly spaced single vacancies were placed in a cubic simulation box containing 215000 silicon atoms arranged in a diamond lattice. The vacancy population was evolved at $1600 \mathrm{~K}$ and zero pressure using velocity rescaling for temperature control. Time integration was performed using the Gear fifth-order predictor-corrector algorithm with a time step of $0.77 \mathrm{fs}$. The environmentdependent interatomic potential (EDIP) ${ }^{13}$ was used to com-

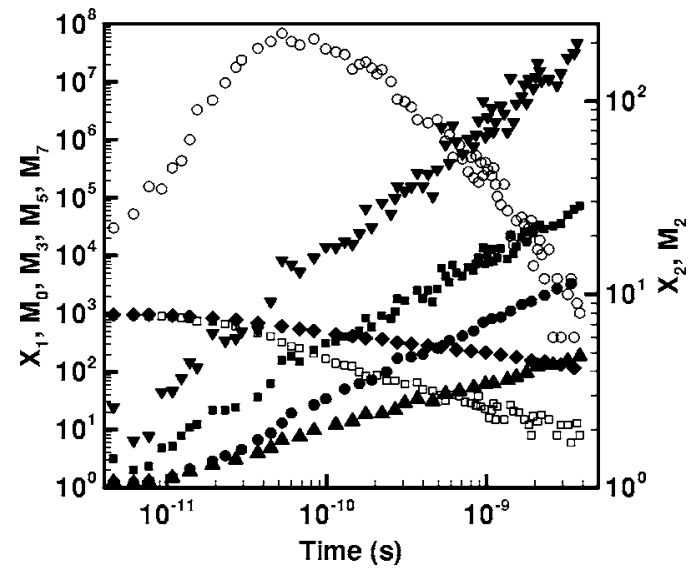

FIG. 5. Various components of the vacancy cluster size distribution. $X_{1}$ is the monomer (open squares); $X_{2}$, dimer (open circles); $M_{0}$, the number of clusters (solid diamonds); $M_{2}$, the average cluster size (solid circles); $M_{3}$, third-order moment (solid deltas); $M_{5}$, fifth-order moment (solid squares); and $M_{7}$, seventh-order moment (solid gradients).

pute the atomic forces. The EDIP has been demonstrated to give excellent predictions ${ }^{19}$ for vacancy and cluster energies and structures.

The size distribution of vacancy clusters was monitored periodically. At each sampling time, the locations of the vacancies were determined by quenching the lattice at constant density and then comparing the atomic coordinates to a reference perfect crystal. The individual vacancies were then assigned to clusters based on their mutual separation according to the Stillinger cluster criterion. ${ }^{21}$ Finally, the clusters were used to compute a size distribution and its moments for use in the regression described below. Various components of the cluster size distribution are shown in Fig. 5. The various moments of the size distribution are defined as $M_{n}$ $=\sum_{s} s^{n} X_{s}$, where $X_{s}$ is the number of clusters of size $s$, and $n$ is the moment order. Of particular note is the statistical quality of the data, which exhibits relatively little scatter due to the large system size.

\section{B. Regression approach}

The regression approach for determining the KMC rate parameters is shown in Fig. 6. For each comparison, the $\mathrm{KMC}$ simulation is initialized in exactly the same manner as the MD calculation described above and evolved for the same duration of time. The input parameters to the KMC simulation are the bond energies and the overall attempt frequency, $v_{0}$. Once the KMC run is completed, a normalized objective function is evaluated based on a least-squares comparison of the KMC and MD size distribution components,

$$
\underset{\nu_{0}, E_{b}^{j}}{O F}=\frac{1}{N} \sum_{i=1}^{N}\left(\frac{2\left(X_{i}^{K M C}-X_{i}^{M D}\right)}{X_{i}^{K M C}+X_{i}^{M D}}\right)^{2},
$$

where $O F$ is the objective function, $\nu_{0}$ is overall attempt frequency, $E_{b}^{j}$ is the bond energy for interaction $j$, and $X_{i}^{K M C}$ and $X_{i}^{M D}$ are the data points at sampling point $i$ from the 


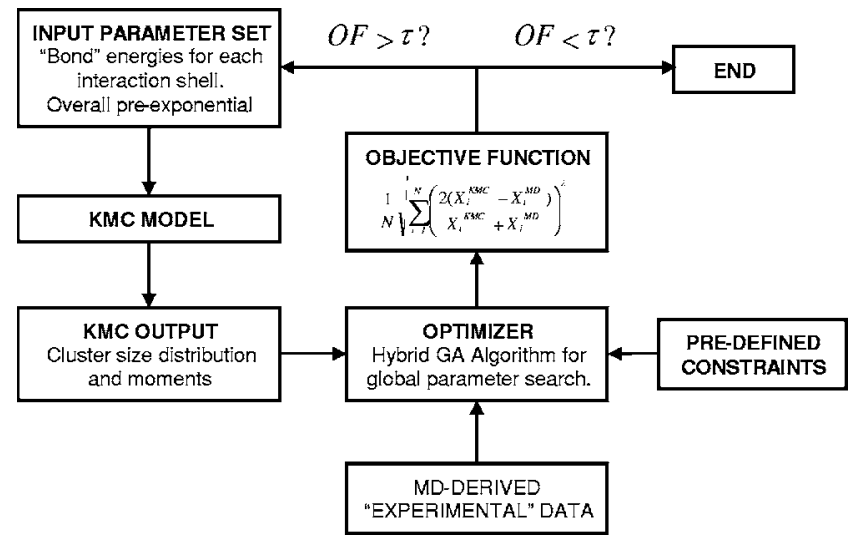

FIG. 6. KMC rate tuning framework.

KMC and MD simulations, respectively. Finally, $N$ is the total number of comparison points used in the regression.

As shown in Fig. 5, included in the comparison are the concentration of monomers and dimers as well as several moments. The motivation for using high-order moments is to emphasize the large cluster contribution to the data given the relatively short evolution time (about $5 \mathrm{~ns}$ ). The results of three KMC runs are averaged at each objective function evaluation to reduce the noise in the objective function.

The optimizer is based on a hybrid genetic algorithm (GA) described in detail in Ref. 22, which allows for a global parametric search on a nonconvex surface. Various constraints are imposed during the optimization process. Some of these constraints are predefined "hard constraints" on the allowable values of the bond energies and attempt frequency to physically reasonable values. Other constraints are implemented as "soft constraints" that add a penalty term to the objective function defined in Eq. (6). Each time the GA generates a new population of individuals, the corresponding sets of parameters are passed to the KMC simulation to obtain new objective function (fitness) values. The ability of the GA to sample local minima is enhanced by including periodic local minimization based on the simplex method. In this approach, the best $N+1$ individuals are used to create an $\mathrm{N}$-dimensional simplex every three to four generations. This simplex is evolved to the local minimum, and the new simplex vertices are then passed back to the GA to replace the worst individual in the current generation. The GA then proceeds to create the next generation using a sequence of mating and mutation operations.

\section{RESULTS AND DISCUSSION}

Two sequences of rate regression studies were performed. In the first, no constraints were applied other than the predefined hard constraints, while in the second, a penalty function was additionally applied to the objective function in Eq. (6). These cases are discussed separately in the following sections.

\section{A. Unconstrained optimization}

A sequence of KMC models, with different interaction ranges, was regressed to the MD data shown in Fig. 5. The

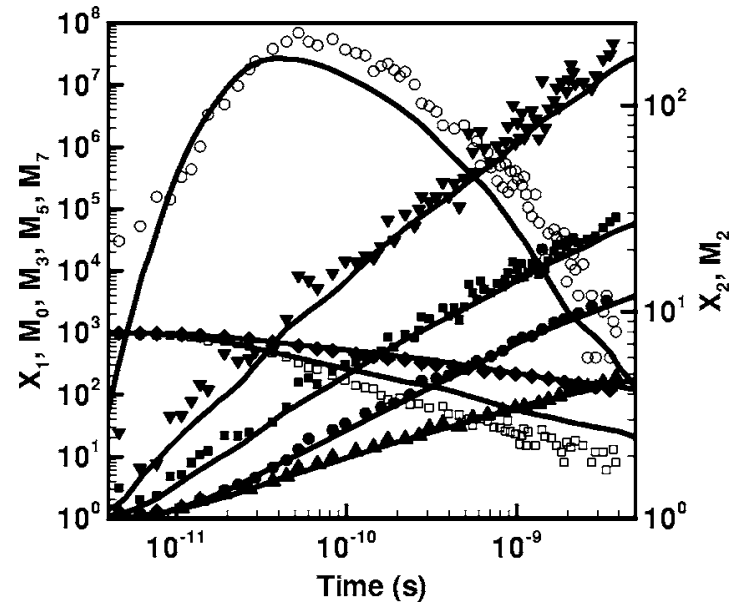

FIG. 7. Comparison of MD and fitted KMC model (2NN) predictions for the vacancy cluster size evolution. Evolution profiles are symbols for MD, solid lines for $\mathrm{KMC} ; X_{1}$ is the monomer (open squares); $X_{2}$, dimer (open circles); $M_{0}$, the number of clusters (solid diamonds); $M_{2}$, the average cluster size (solid circles); $M_{3}$, thirdorder moment (solid deltas); $M_{5}$, fifth-order moment (solid squares); and $M_{7}$, seventh-order moment (solid gradients).

interaction distance in these models ranged from $2 \mathrm{NN}$ up to $8 \mathrm{NN}$, and the screening physics discussed in Sec. II B was activated in all cases. For each fit, the bond energies and as well as the overall attempt frequency, $\nu_{0}$, were allowed to vary subject to the predefined constraints. Note that the number of fitting parameters increases proportionately to the vacancy-vacancy-interaction distance. The fit quality (i.e., the agreement between the KMC and MD cluster size distributions) was generally found to increase slightly with increasing interaction range, although almost all models led to good representations of the MD data; the results for $2 \mathrm{NN}$ and 8NN KMC model fits are shown in Figs. 7 and 8, respectively. Except for the monomer concentration evolution being somewhat slow in the $2 \mathrm{NN}$ case, both fits are excellent overall.

As expected, the computational effort required to find the best parameter values increased somewhat with increasing number of parameters, and for the largest interaction distances it was not possible to be certain that the very best parameter sets possible were located by the time the optimization was terminated. The uncertainty was minimized using several regression runs with different initial conditions. Termination of the regression in each case was determined by monitoring the evolution of the best objective function, and typically about 8000 to 10000 function evaluations were required before the objective function stopped evolving. In all cases, the statistical fluctuations in both the MD and KMC results also resulted in more difficult global optimizations. The best objective functions obtained for each KMC model are shown in Table I.

Also shown in Table I for each run is the ratio of the KMC single vacancy diffusivity, $D_{V 1}^{K M C}$, to the MD value, $D_{V 1}^{M D}$. Recall that the single vacancy diffusivity is given by $D_{V 1}=v_{0} \exp \left(-\Delta E_{h o p} / k T\right)$ and is proportional to the overall attempt frequency. Interestingly, as the interaction range increases from $2 \mathrm{NN}$ to $8 \mathrm{NN}$, the ratio decreases almost linearly 


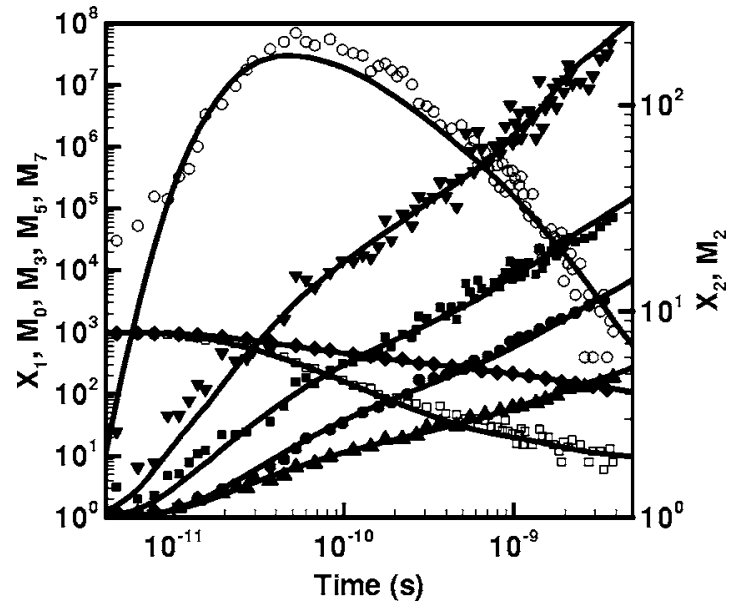

FIG. 8. Comparison of MD and fitted KMC model (8NN) predictions for the vacancy cluster size evolution. Evolution profiles are symbols for $\mathrm{MD}$, solid line for $\mathrm{KMC} ; X_{1}$ is the monomer (open squares); $X_{2}$, dimer (open circles); $M_{0}$, the number of clusters (solid diamonds); $M_{2}$, the average cluster size (solid circles); $M_{3}$, thirdorder moment (solid deltas); $M_{5}$, fifth-order moment (solid squares); and $M_{7}$, seventh-order moment (solid gradients).

from 2.4 to unity. In other words, the best objective function does not improve significantly as the interaction distance increases because the regression process artificially increases the overall attempt frequency in the system to compensate for the incorrect physics. It is notable that the correct diffusivity is almost exactly reproduced once the correct interaction distance $(8 \mathrm{NN})$ is specified. Also note that this information is not explicitly provided in any constraint, but rather is implicitly present in the cluster size distribution evolution. The vacancy diffusivity ratio evolution with interaction distance is shown graphically in Fig. 9, and other than the scatter in the $6 \mathrm{NN}$ point, the regressed diffusivity ratio appears to evolve smoothly to unity at $8 \mathrm{NN}$.

\section{B. Constrained optimization}

The same series of experiments described in the previous section was repeated, but this time the overall attempt frequency was constrained so that the KMC single vacancy diffusivity was matched to the MD value during the optimiza-

TABLE I. Objective function values and vacancy diffusivity ratios for KMC models with different interaction ranges. Unconstrained regression.

\begin{tabular}{ccc}
\hline \hline Model & Objective function & $\mathrm{D}_{\mathrm{V} 1}^{\mathrm{KMC}} / \mathrm{D}_{\mathrm{V} 1}^{\mathrm{MD}}$ \\
\hline $2 \mathrm{NN}$ & 0.0741 & 2.37 \\
$3 \mathrm{NN}$ & 0.0257 & 2.27 \\
$4 \mathrm{NN}$ & 0.0214 & 2.05 \\
$5 \mathrm{NN}$ & 0.0239 & 1.81 \\
$6 \mathrm{NN}$ & 0.0361 & 1.15 \\
$7 \mathrm{NN}$ & 0.0157 & 1.25 \\
$8 \mathrm{NN}$ & 0.0211 & 1.05 \\
\hline \hline
\end{tabular}

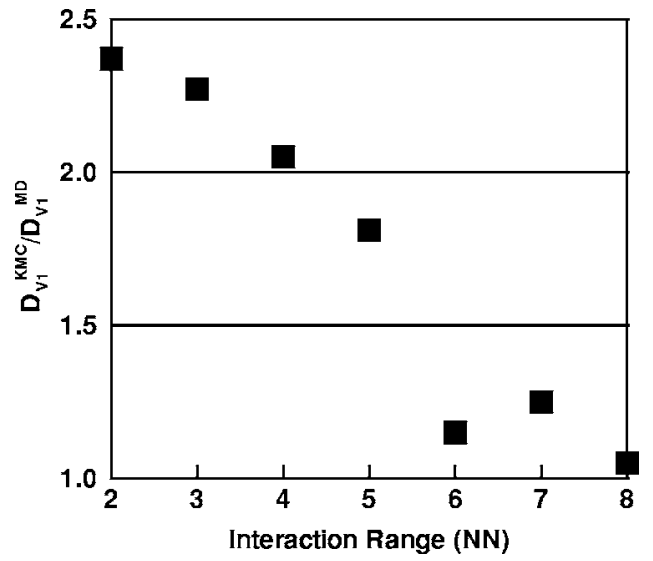

FIG. 9. Ratio of single vacancy diffusivity obtained from KMC models and MD as a function of KMC model interaction distance.

tion. This constraint was implemented by adding a penalty term to the objective function in Eq. (6). The optimization problem is now formulated as

$$
\underset{\nu_{0}, E_{b}^{j}}{O F}=\frac{1}{N} \sum_{i=1}^{N}\left(\frac{2\left(X_{i}^{K M C}-X_{i}^{M D}\right)}{X_{i}^{K M C}+X_{i}^{M D}}\right)^{2}+K\left(D_{V 1}^{K M C}-D_{V 1}^{M D}\right)^{2}
$$

where $K$ is the penalty constant, which was set at 5 for all runs.

The resulting best objective functions and diffusivity ratios are shown in Table II. Because of the constraint on the single vacancy diffusivity, the objective function values now decrease monotonically with increasing the interaction range, and the improvement of the fitting with increasing interaction range is now significant as shown in Figs. 10 and 11 for the constrained $2 \mathrm{NN}$ and $8 \mathrm{NN}$ KMC models, respectively. In other words, the $2 \mathrm{NN}$ fit is now demonstrably worse than the $8 \mathrm{NN}$ fit, showing that it is impossible to obtain the correct evolution while restricting the monomer diffusivity to its MD value. As shown in Table II, forcing the KMC single vacancy diffusivity to the MD value results in lower ratios at each interaction distance, but once again a ratio of unity is not attained until the correct interaction range is used in the KMC simulation. Finally, the constrained and unconstrained regressions give very similar fits to the MD data both in

TABLE II. Objective function values and vacancy diffusivity ratios for KMC models with different interaction ranges. Constrained regression.

\begin{tabular}{ccc}
\hline \hline Model & Objective function & $\mathrm{D}_{\mathrm{V} 1}^{\mathrm{KMC}} / \mathrm{D}_{\mathrm{V} 1}^{\mathrm{MD}}$ \\
\hline $2 \mathrm{NN}$ & 0.5415 & 1.47 \\
$3 \mathrm{NN}$ & 0.2668 & 1.31 \\
$4 \mathrm{NN}$ & 0.1642 & 1.18 \\
$5 \mathrm{NN}$ & 0.1274 & 1.15 \\
$6 \mathrm{NN}$ & 0.0515 & 1.06 \\
$7 \mathrm{NN}$ & 0.089 & 1.01 \\
$8 \mathrm{NN}$ & 0.111 & 0.97 \\
\hline \hline
\end{tabular}




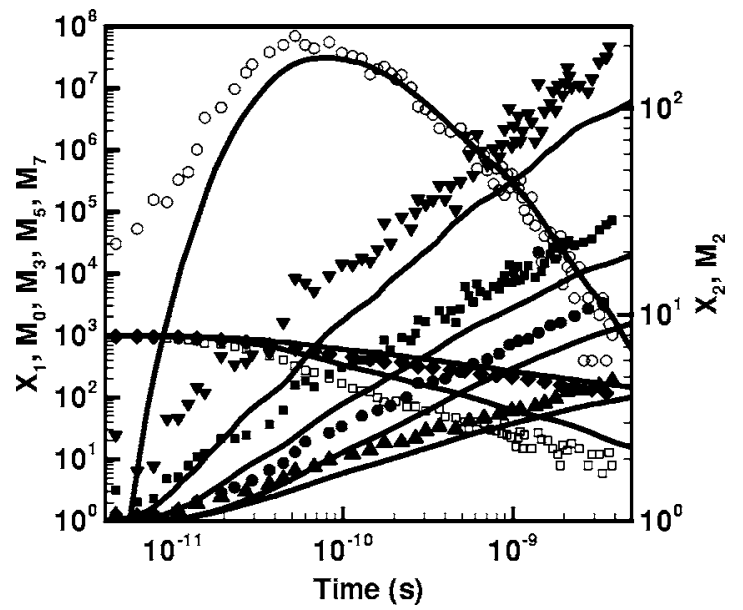

FIG. 10. Comparison of MD and fitted KMC model (2NN) predictions for the vacancy cluster size evolution. Evolution profiles are symbols for MD, solid line for $\mathrm{KMC} ; X_{1}$ is the monomer (open squares); $X_{2}$, dimer (open circles); $M_{0}$, the number of clusters (solid diamonds); $M_{2}$, the average cluster size (solid circles); $M_{3}$, thirdorder moment (solid deltas); $M_{5}$, fifth-order moment (solid squares); and $M_{7}$, seventh-order moment (solid gradients).

terms of quality and parameter values, further demonstrating the robustness of the approach.

\section{Unscreened interactions}

To assess the effect of the screening physics introduced in Sec. II B, several of the KMC models with the screening physics deactivated were regressed to the MD evolution data in Fig. 5 without any constraint on the overall attempt frequency. None of the optimizations were found to give a satisfactory representation of the MD data. The predictions of the optimized 6NN KMC model, which led to the lowest

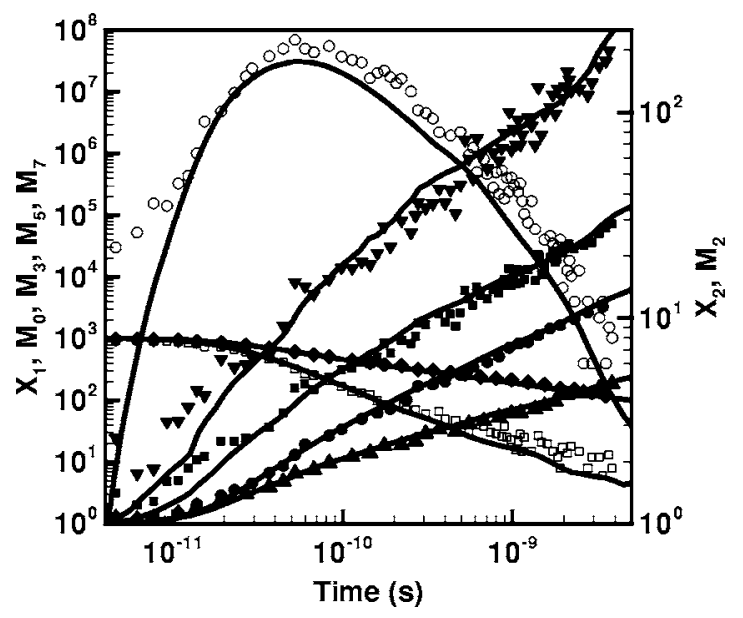

FIG. 11. Comparison of MD and fitted KMC model (8NN) predictions for the vacancy cluster size evolution. Evolution profiles are symbols for MD, solid line for KMC; $X_{1}$ is the monomer (open squares); $X_{2}$, dimer (open circles); $M_{0}$, the number of clusters (solid diamonds); $M_{2}$, the average cluster size (solid circles); $M_{3}$, thirdorder moment (solid deltas); $M_{5}$, fifth-order moment (solid squares); and $M_{7}$, seventh-order moment (solid gradients).

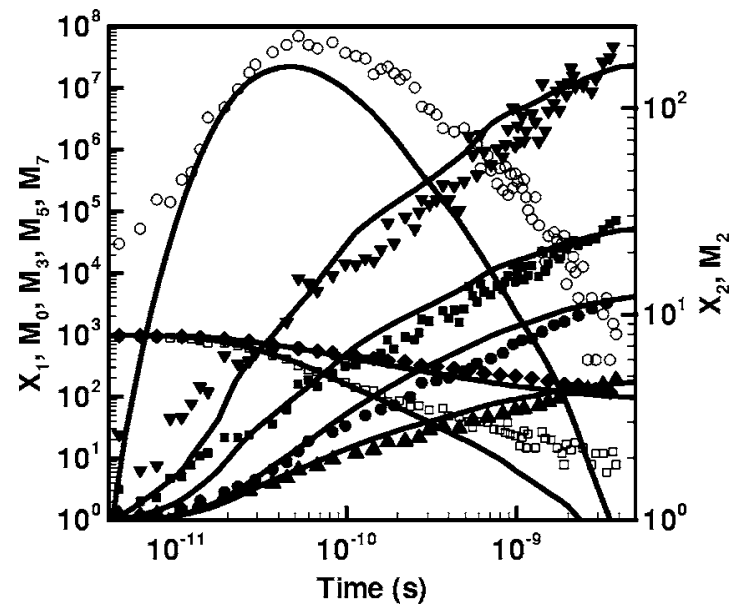

FIG. 12. Comparison of MD and fitted KMC model (with no screening) predictions for the vacancy cluster size evolution. Evolution profiles are symbols for MD, solid line for $\mathrm{KMC} ; X_{1}$ is the monomer (open squares); $X_{2}$, dimer (open circles); $M_{0}$, the number of clusters (solid diamonds); $M_{2}$, the average cluster size (solid circles); $M_{3}$, third-order moment (solid deltas); $M_{5}$, fifth-order moment (solid squares); and $M_{7}$, seventh-order moment (solid gradients).

objective function, are shown in Fig. 12. Clearly the agreement is poor for each component of the cluster size distribution, and provides strong evidence for the relevance of the screening physics. Once again, the effect of the screening physics is to ensure that the void energies scale with the surface area of the clusters, rather than the volume. This result demonstrates that the regressions are not overspecified, i.e., even with nine parameters, it is still not possible to obtain good agreement unless the model physics are correct.

\section{Cluster morphologies and the effect of entropy}

The inclusion of the full interaction range $(8 \mathrm{NN})$ in the KMC model has profound effects on the predicted cluster morphologies. Shown in Figs. 13(a) and 13(b) are snapshots at $5 \mu$ s of the overall vacancy distribution taken from $2 \mathrm{NN}$ and $8 \mathrm{NN}$ KMC simulations, respectively. In the former, the clusters are observed to exhibit highly compact, faceted structures. The facets are aligned along (111) orientations as observed experimentally for much larger voids found in Czochralski silicon crystals after cooling. ${ }^{8}$ In other words, these structures are close to their expected energetic ground states.

On the other hand, the clusters in the $8 \mathrm{NN}$ simulation are highly amorphous, extended structures, which are similar to those found in MD simulations. Keep in mind that the clusters in Fig. 13(b) are, of course, on-lattice structures because of the fixed KMC lattice, while in MD simulations the "real" clusters are best described as relatively amorphous regions that contain the same number of vacancies. The extended clusters in Fig. 13(b) have a larger capture radius, and are more mobile, than the compact structures in Fig. 13(a). These two features lead to an enhancement of the evolution dynamics in agreement with the MD predicted behavior. Representative clusters from each simulation are shown in Fig. 14. 


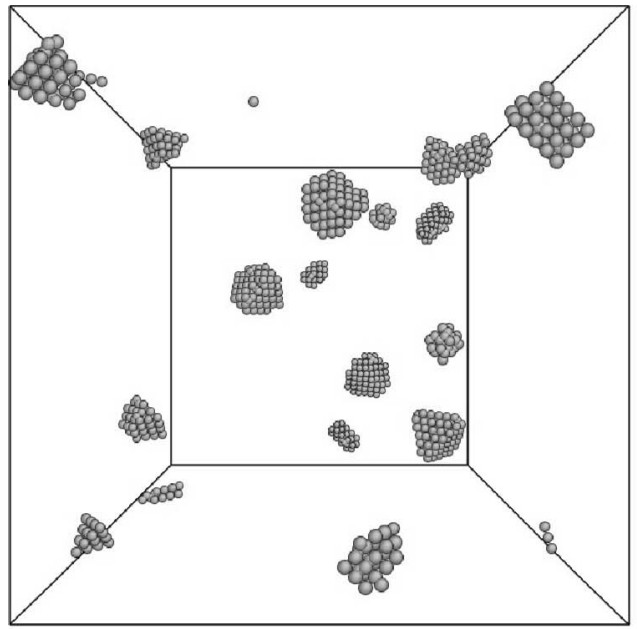

(a)

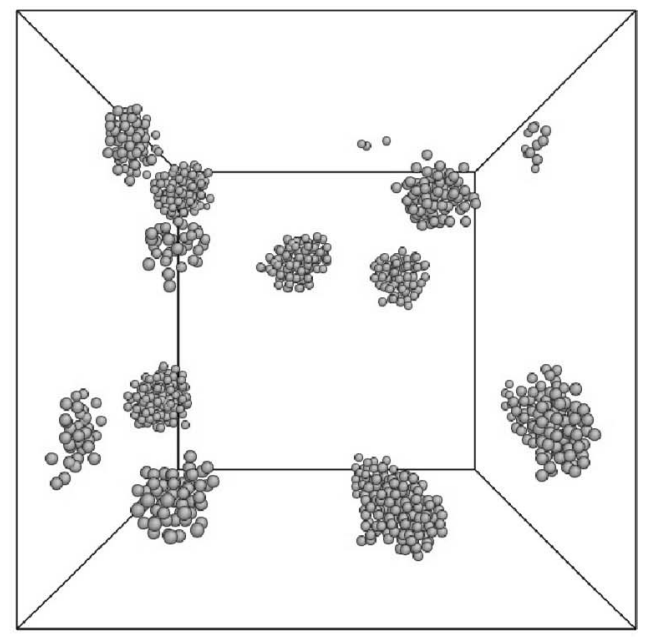

(b)

FIG. 13. Snapshots of vacancy cluster distributions at $5 \mu$ s; (a) 2NN model and (b) 8NN model.

The difference between the cluster morphologies in the 2NN and 8NN KMC models can best be understood by considering the thermodynamics of the real, continuous system. We have recently shown that the high temperature properties of atomic clusters in silicon are dominated by the presence of an extremely large number of mechanically stable off-lattice configurations. These states lead to significant configurational entropy, which has been neglected in the literature, and in conjunction with the vibrational entropy, shift the free energy minimum from the energetic ground state to a collection of extended structures such as the one shown in Fig. 14(b). Interestingly, EDIP MD simulations at $1600 \mathrm{~K}$ show vacancy cluster morphologies that are qualitatively similar to the one shown in Fig. 14(b). A more direct comparison is not possible because the MD structures quench into complex offlattice configurations that make vacancy identification ambiguous.

The physics of the $2 \mathrm{NN}$ and $8 \mathrm{NN}$ KMC models were further probed by computing cluster diffusion coefficients. Note that, like the single vacancy diffusivity, cluster diffu-

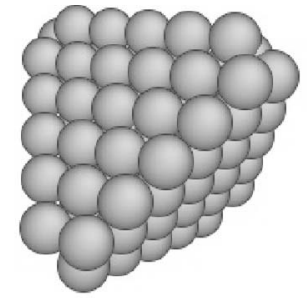

(a)

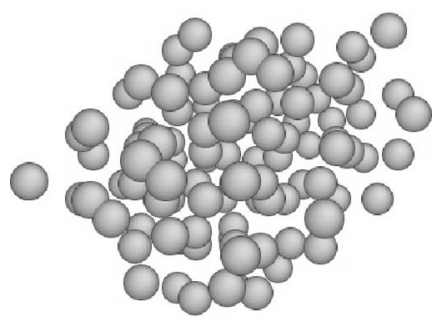

(b)

FIG. 14. Comparison of cluster morphologies predicted by (a) $2 \mathrm{NN}$ model and (b) 8NN model.

sivities are not explicitly considered in the regression process. Furthermore they are complex functions of many types of single vacancy hops with different transition rates. As a result they provide a strong test of the overall physical robustness of the KMC models generated in this work. Shown in Fig. 15 are various cluster diffusion coefficients predicted by the $2 \mathrm{NN}$ and $8 \mathrm{NN}$ KMC models, along with the values computed with full MD. Details of the calculation approach used to compute the diffusion coefficients are given in Ref. 11. The agreement between the 8NN KMC model predictions and the MD values is excellent for all cluster sizes (including single vacancies). On the other hand, the $2 \mathrm{NN}$ KMC model leads to substantially worse representation of the cluster diffusivities. The regression procedure artificially inflates the value of the overall attempt frequency to com-

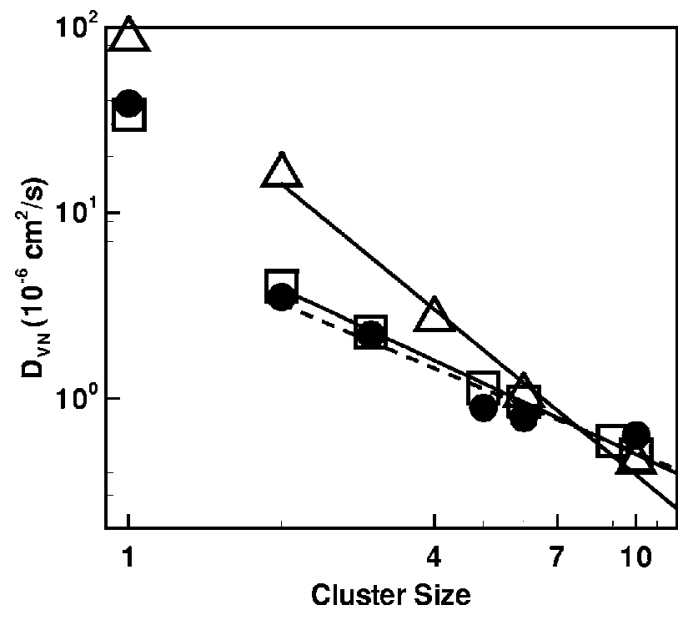

FIG. 15. Comparison of cluster diffusivities from $2 \mathrm{NN}$ and $8 \mathrm{NN}$ $\mathrm{KMC}$ models. Solid line and open squares are MD values; dashed line and solid circles, 8NN KMC values; solid line and open deltas, 2NN KMC values. 
pensate for the low mobility of the compact structures. As a result, small clusters are too fast, while larger ones become too slow.

The ability of the regression approach presented in this work to correctly reproduce the MD dynamics, while predicting the correct vacancy cluster diffusion coefficients and cluster morphologies, implies that we have correctly projected the full dimensional thermodynamics onto the restricted subspace of the lattice KMC. In some sense, clusters such as the one shown in Fig. 14(b) are representative structures of ensembles of complex off-lattice morphologies that appear in MD simulations. On the other hand, restricting the cluster definition to the $2 \mathrm{NN}$ interaction distance greatly reduces the degrees of freedom available to capture this effect and leads to compact structures, which are not observed in EDIP MD simulations at $1600 \mathrm{~K}$.

\section{E. KMC evolutions based on on-lattice energies}

Our final investigation was to assess the standard approach of computing energy barriers using the energies of on-lattice configurations and Eqs. (3) and (4). A very large number of on-lattice structures $(\sim 10000)$ were generated and statically relaxed with conjugate gradient energy minimization based on the EDIP potential. The structure-energy database was then used to extract "on-lattice bond energies" up to $8 \mathrm{NN}$. The regressed bond energies were found to almost exactly reproduce the MD formation energy of all structures in the database. Note that the lattice-based barriers still capture some elements of the interatomic potential because of the static relaxation used to compute the configuration energies.

The resulting "on-lattice" bond energies were then used to compute energy barriers in a new 8NN KMC simulation, in which the overall attempt frequency was fixed to the MD value. The predicted KMC evolution is compared to the MD data in Fig. 16 and exhibits substantially slower evolution. In fact, shortly after most of the monomers are consumed at about $300 \mathrm{ps}$, the evolution is dramatically slowed because of the high-energy barriers associated with cluster breakup and rearrangement, which are required for diffusion and coalescence. It was found that the overall attempt frequency needed to be increased about 20 -fold before the evolution became comparable to the MD rate.

The failure of this model to predict even qualitatively reasonable evolution can be attributed to the fact that in this approach the on-lattice structures do not represent in any way the physics of the real off-lattice structures. In particular, the configurational and vibrational entropy associated with the off-lattice ensemble is completely neglected. Note that the on-lattice configurational entropy naturally is included in any KMC model, but as we have shown in other work, ${ }^{20}$ it is not sufficient to lead to destabilization of the compact geometry at any temperature of interest.

\section{CONCLUSIONS}

A regression approach was used to compute effective bond energies for vacancy clusters in an on-lattice KMC

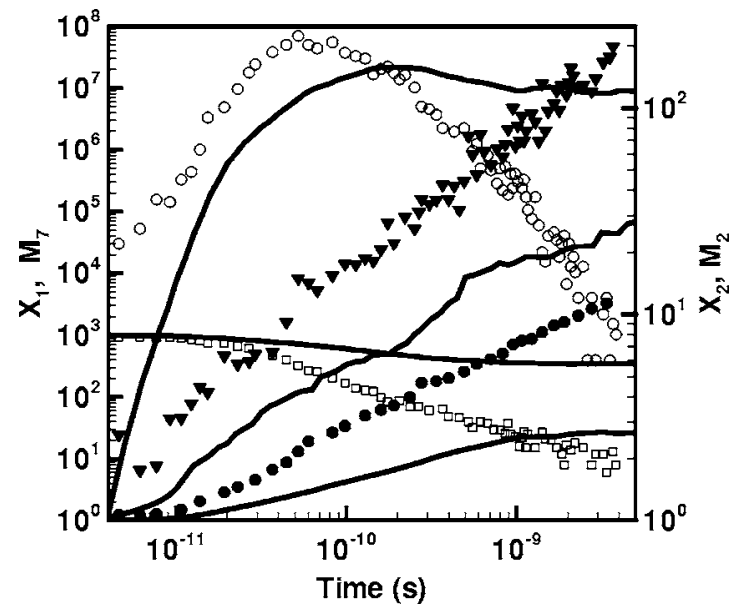

FIG. 16. Comparison of MD and on-lattice energy KMC model (with interaction screening) predictions for the vacancy cluster size evolution. Evolution profiles are symbols for MD, solid line for $\mathrm{KMC} ; X_{1}$ is the monomer (open squares); $X_{2}$, dimer (open circles); $M_{2}$, the average cluster size (solid circles); and $M_{7}$, seventh-order moment (solid gradients).

model. The resulting bond energies lead to an excellent representation of vacancy clustering at high temperature. It is shown that two elements are required to capture the correct physics. The first is a large vacancy-vacancy interaction distance, up to the eighth neighbor shell, while the second is a correct projection of continuous-space entropic effects onto the lattice subspace of the KMC simulation. In the present work, these entropic contributions were obtained indirectly by the regression to MD evolution data. A more direct connection between the lattice and continuous spaces will be the subject of future work.

Some limitations of the present approach should be noted. First, the MD evolution data is necessarily limited to very small time scales (ns). This means that atomic processes that occur on slower time scales are not captured in the MD data and therefore are not transmitted to the KMC rate parameters. The high temperature used in the present work mitigates some of these limitations for the specific case of vacancy aggregation. The fact that the predicted cluster diffusion coefficients and morphologies are in excellent agreement with MD values is a strong indicator that the essential components of the problem physics are captured. In other words, vacancy cluster diffusion and coalescence occur by a sequence of single-vacancy events that are captured fully by the MD data. A second limitation of the approach is the lack of transferability to different temperatures. The $\mathrm{KMC}$ bond energies regressed in this study are valid only at $1600 \mathrm{~K}$ because they contain temperature dependent information from the full continuous-space problem, such as vibrational and configurational entropies. Unless these entropies are explicitly known, there is no way to rescale the parameters for use at other temperatures without repeating the entire regression process. Future work will be aimed at estimating explicitly these contributions to generalize the approach to variable temperature situations, and remove the restriction posed by the need for multiple lengthy large-scale MD simulations. 
The above limitations notwithstanding, it is expected that the approach presented in this paper for developing KMC models should be useful for a variety of systems that are accessible with direct MD simulation. KMC modeling of the evolution of defect clusters in multicomponent semiconductor systems such as silicon-germanium and silicon-carbide, in particular, potentially requires the specification of an enormous number of event rates because of the large number of different microconfigurations possible. With the present approach, many of the redundant transitions would be lumped together automatically, the precise nature of the lumping being dependent on the specific KMC rate kernel being param- etrized. Similarly complex aggregation processes in metallic systems, such as point defect-impurity coaggregation, also should be amenable to treatment within the current framework.

\section{ACKNOWLEDGMENTS}

We gratefully acknowledge financial support from the National Science Foundation (CTS01-34418) and the Department of Chemical and Biomolecular Engineering at the University of Pennsylvania.
*Email address: talid@seas.upenn.edu

${ }^{1}$ M. E. Law, G. H. Gilmer, and M. Jaraiz, MRS Bull. 25, 45 (2000).

${ }^{2}$ J. B. Adams, Z. Y. Wang, and Y. H. Li, Thin Solid Films 365, 201 (2000).

${ }^{3}$ R. Pinacho, P. Castrillo, M. Jaraiz, I. Martin-Bragado, J. Barbolla, H.-J. Gossmann, G. H. Gilmer, and J. L. Benton, J. Appl. Phys. 92, 1582 (2002).

${ }^{4}$ O. Biham, I. Furman, M. Karimi, G. Vidali, R. Kennett, and H. Zeng, Surf. Sci. 400, 29 (1998).

${ }^{5}$ L. G. Wang and P. Clancy, Surf. Sci. 473, 25 (2001).

${ }^{6}$ A. La Magna, S. Coffa, and L. Colombo, Nucl. Instrum. Methods Phys. Res. B 148, 262 (1999).

${ }^{7}$ K. A. Fichthorn and M. Scheffler, Phys. Rev. Lett. 84, 5371 (2000).

${ }^{8}$ M. Itsumi, H. Akiya, T. Ueki, M. Tomita, and M. Yamawaki, J. Appl. Phys. 78, 5984 (1995).

${ }^{9}$ E. Dornberger, Ph.D. Thesis, Universite Catholique de Louvain, Belgium, 1998.
${ }^{10}$ T. Sinno and R. A. Brown, J. Electrochem. Soc. 146, 2300 (1999).

${ }^{11}$ M. Prasad and T. Sinno, Phys. Rev. B 68, 045207 (2003).

${ }^{12}$ T. Frewen, S. S. Kapur, W. Haeckl, W. von Ammon, and T. Sinno, J. Cryst. Growth 279, 258 (2005).

${ }^{13}$ M. Z. Bazant, E. Kaxiras, and J. F. Justo, Phys. Rev. B 56, 8542 (1997).

${ }^{14}$ J. Tersoff, Phys. Rev. B 37, 6991 (1988).

${ }^{15}$ F. H. Stillinger and T. A. Weber, Phys. Rev. B 31, 5262 (1985).

${ }^{16}$ A. Bongiorno, L. Colombo, F. Cargnoni, C. Gatti, and M. Rosati, Europhys. Lett. 50, 608 (2000).

${ }^{17}$ A. Bongiorno and L. Colombo, Phys. Rev. B 57, 8767 (1998).

${ }^{18}$ M. Prasad and T. Sinno, Phys. Rev. B 68, 045206 (2003).

${ }^{19}$ M. Prasad and T. Sinno, Appl. Phys. Lett. 80, 1951 (2002).

${ }^{20}$ S. S. Kapur, M. Prasad, J. C. Crocker, and T. Sinno, Phys. Rev. B 72, 014119 (2005).

${ }^{21}$ F. H. Stillinger, J. Chem. Phys. 38, 1486 (1963).

${ }^{22}$ T. A. Frewen, T. Sinno, W. Haeckl, and W. von Ammon, Comput. Chem. Eng. 29, 713 (2005). 